\title{
Investigation on the mental health status of pregnant women in China during the Pandemic of COVID-19
}

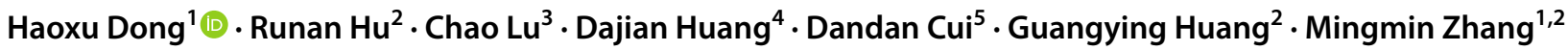

Received: 6 May 2020 / Accepted: 16 September 2020 / Published online: 3 October 2020

(c) Springer-Verlag GmbH Germany, part of Springer Nature 2020

\begin{abstract}
Purpose To evaluate the anxiety and depression in pregnant women in China, and its influencing factors during the corona virus disease 2019 (COVID-19) pandemic.

Methods From February 22 to February 27, a questionnaire survey was conducted on 156 pregnant women, including demographic characteristics, a self-rating anxiety scale (SAS), and a self-depression rating scale (SDS).

Results A total of 13 non-homologous end-joining $(8.3 \%, 13 / 156)$ patients were anxious, 79 patients $(50.6 \%, 79 / 156)$ were depressed, and 13 patients $(8.3 \%, 13 / 156)$ suffered from both anxiety and depression. The SAS score of pregnant women was $40.55 \pm 6.09$, and the SDS score was $50.42 \pm 11.64$. For the SAS score, only $8.3 \%$ of all patients $(13 / 156)$ were in a light anxiety state. For the SDS score, $46.79 \%$ (73/156) of patients was normal, $23.72 \%$ of patients (37/156) showed mild depression, 22.44\% (35/156) showed moderate depression, and 4.49\% (7/156) showed severe depression. No significant changes were observed in SAS and SDS scores between patients from different regions within China, health state, gestational week, educational background, and living condition $(P>0.05)$. Moreover, no significant differences were observed between diagnosed/suspected patients and the normal control group $(P>0.05)$, and between pregnant women in Wuhan compared to other regions $(P>0.05)$.

Conclusion During the COVID-19 epidemic, the anxiety level of pregnant women was the same as that before the epidemic, while the level of depression was significantly higher. Pregnant women who lived in Wuhan, the epicenter of the epidemic, were not more anxious or depressed compared to pregnant women in other regions during the COVID-19 epidemic. Furthermore, the mental health status of pregnant women with COVID-19 was not more severe.
\end{abstract}

Keywords COVID-19 $\cdot$ Pregnant women $\cdot$ Mental health $\cdot$ Anxiety $\cdot$ Depression

Electronic supplementary material The online version of this article (https://doi.org/10.1007/s00404-020-05805-x) contains supplementary material, which is available to authorized users.

Mingmin Zhang

mmzhang@tjh.tjmu.edu.cn

1 Department of Traditional Chinese Medicine, Tongji Hospital, Tongji Medical College, Huazhong University of Science and Technology, Jiefang Road 1095\#, Wuhan 430030, Hubei Province, China

2 Institute of Integrated Traditional Chinese and Western Medicine, Tongji Hospital, Tongji Medical College, Huazhong University of Science and Technology, Jiefang Road 1095\#, Wuhan 430030, Hubei Province, China

3 Department of Traditional Chinese Medicine, Yichang Second People's Hospital, Xiling First Road 21\#, Yichang, Hubei Province, China

\author{
Abbreviations \\ COVID-19 Corona virus disease 2019 \\ SAS Self-rating anxiety scale \\ SDS Self-depression rating scale
}

4 Department of Adult Internal Medicine, Maternal and Child Hospital of Hubei Province, Tongji Medical College, Huazhong University of Science and Technology, Wuluo Road 745\#, Wuhan, Hubei Province, China

5 Department of Traditional Chinese Medicine, Union Hospital, Tongji Medical College, Huazhong University of Science and Technology, Jiefang Road 1277\#, Wuhan, Hubei Province, China 


\section{Introduction}

Corona virus disease 2019 (COVID-19), which has strong infectivity and severe pathogenicity [1], first broke out in Wuhan, China in December 2019, and has been a threat to the health of the people all over the world. It has since affected over 180 countries and had a major outbreak in many countries, such as Italy, the United Kingdom, Spain, France, Russia, South Korea, Iran, Brazil, India, the United States, and more [2]. Until August 24, 2020, a cumulative total of over 23 million cases and 800000 COVID-19 related deaths have been reported.[3].

Wuhan, the capital of the Hubei Province, has close links with surrounding cities by countless traffic lines, and has been considered the center of the first stage of the COVID-19 epidemic in China. On January 22, 2020, Wuhan locked down the entire city, and adopted a series of strict restrictions to prevent the further spread of COVID19. Restrictions included closing inter-city transportation and intra-city public transportation, restricting free access outside communities, and recommending everyone to stay at home. Other cities in the Hubei Province gradually adopted the same restrictions as Wuhan, and the other provinces adopted relatively mild restrictions. In China, about $60.8 \%$ of confirmed cases were located in Wuhan and $82.4 \%$ were located in the Hubei Province [2]. Since January 27, 2020, the number of new cases per day was over 1000, and on February 12, it reached the peak and at least 15,152 cases were confirmed. Since February 22, the number of cases had never been more than 648 [2]. From March 6, the number of confirmed cases started to drop below 100 [2].

An epidemic may cause psychological pressure on pregnant women living in the epidemic area. From previous studies on severe acute respiratory syndrome (SARS), which is related to COVID-19, pregnant women were more likely to have an adverse pregnancy outcome when compared to women who were not pregnant [4-8]. Therefore, it is thought that pregnant women are more susceptible to COVID-19 than the general population and deserve more attention. During the COVID-19 pandemic, a series of restrictions have been taken in China, especially in Wuhan, the center of the outbreak. Therefore, it may make be difficult for pregnant women to receive routine obstetric examinations, and the treatment of pregnant women with COVID-19 may be complicated. Considering the above-mentioned factors, COVID-19 may likely affect the mental health of pregnant women. Until now, many other studies presented conflicting conclusions about the overall increase or non-increasing incidence of post-traumatic stress disorder caused by depression and different disasters [9-12]. In a recently published cross-sectional study, it was found that compared with women who were hospitalized before the COVID-19 pandemic, women who were hospitalized in high-risk pregnancy wards during the strict quarantine period of COVID-19 did not show an increased risk of depression [13]. This study was carried out to investigate the mental health status of pregnant women in China during the COVID-19 pandemic.

\section{Methods}

\section{Recruitment and participants}

The main participants of this study were pregnant women in China. To compare the mental health status of pregnant women in different regions within China during the COVID-19 pandemic, questionnaires in Wuhan, other cities of the Hubei province, and other provinces in China were collected. This study was approved by the Medical Ethics Committee of Tongji Hospital, Tongji Medical College, Huazhong University of Science and Technology, Wuhan, China (Approval number: TJ-IRB20200323).

\section{Study design}

Questionnaires about mental health were designed, and included questions about background of the pregnant woman, including age, educational background, current gestational weeks, themselves or family being infected by COVID-19, region (in Wuhan, in Hubei but not Wuhan, in China but not in the Hubei province), living conditions (alone or not). Then, pregnant volunteers were asked to finish the SAS scares (self-rating anxiety scale [14]) and SDS scares (self-rating depression scale [15]) based on their recent feelings. SAS and SDS scares are widely used for assessing an individual's mental state, were designed by Zung et al. and recommended by the United States Department of Education, Health, and Welfare [14-17]. Both the SAS and SDS scale included 20 questions about psychotic emotional symptoms, psychomotor disturbance, somatic disorder, and mental disorders of anxiety or depression. For answering each question, volunteers select the option that best describes their mental state. Each question is scored on a Likert-type scale of 1-4 or reverse scoring ("a little of the time", "some of the time," "a good part of the time", and "most of the time"). The raw total scores were obtained by summarizing the total scores of the 20 questions, and were converted to percentile standard scores. Both the SAS and SDS scales are shown in supplementary Table 1 and supplementary Table 2 .

The questionnaires on mental health were issued through the online WeChat platform from February 22 to February 27 . In addition, partial questionnaires were distributed 
by doctors working in the fever ward or fever clinic with a QR code to the workplace. Researchers introduced the significance of the survey and guided subjects to complete the questions that were difficult to understand. All subjects orally agreed to participate in the study before completing the questionnaire. The questionnaire was designed and published on the Questionnaire website (https://www.wjx. $\mathrm{cn}$ ), and the original data was available on this website (ID: 59,179,377). Data was collected by Chao Lu, Dajian Huang, and Dandan Cui, and were analyzed by Runan Hu.

After being informed of the sponsors, study purpose, method of participation, and the anonymous way of participating in the questionnaire, the first question asked the patient's their willingness to participate in the study: "Are you willing to participate in the study and cooperate with the completion of this Survey?" (a) Yes, please continue the survey. (b) No.Subjects who agreed to participate in the study continued and completed the study.

No private information, such as name and street address was collected. In addition, no blood samples, tissues, cells, and other biological materials were collected. For all subjects, the results were obtained after they completed the questionnaire. All subjects were informed that their answers would be used for publishing purposes.

In total, 156 subjects agreed to participate in the study, and finished the questionnaires, and all questionnaires were completed. Among them, there were 101 questionnaires from pregnant women in Wuhan; 26 questionnaires from other cities of the Hubei province, except Wuhan; and 26 questionnaires from other provinces in China, except Hubei. Furthermore, there were 11 cases of pregnant women with COVID-19 and 1 suspicious case, whose nucleic acid test was negative but the CT lung screening showed a possible infection.

\section{Inclusion and exclusion criteria}

Inclusion criteria were as follows: (1) Pregnant women in China; (2) Pregnant women who are willing to complete the survey; (3) Pregnant women who agreed to participate in this study.

Exclusion criteria were as follows: (1) Women who were not pregnant; (2) Women who had a spontaneous abortion or embryonic abortion during the time of the study; (3) Pregnancy with severe complications; (4) Women who were diagnosed with a mental illness before pregnancy.

\section{Outcome measures}

The main outcome measures of the study included the SAS and SDS score. If the SAS or SDS score was less than or equal to 50 , the volunteer would be defined as "normal". The secondary outcome measures included age, educational background, region (in Wuhan, in Hubei but not Wuhan, in China but not in the Hubei province), and gestational week.

\section{Sample size calculation}

In a previous study, it was shown that the incidence of anxiety and depression in Chinese women during pregnancy was $10 \%$ and $3.5 \%$, respectively [18]. A $10 \%$ increase in the incidence of anxiety and depression during pregnancy was defined as a significant increase in the probability of anxiety or depression during the COVID-19 epidemic. The sample sizes of anxiety and depression were calculated separately, and the larger size was taken as the sample size for this study. The calculated sample size was 95 . Therefore, and considering that about $5 \%$ of questionnaires may be invalid, a total of 100 questionnaires were collected from pregnant women in Wuhan. Furthermore, 25 questionnaires from nonWuhan cities in the Hubei Province and 25 questionnaires from non-Hubei provinces served as control.

\section{Statistical methods}

Data was analyzed by SPSS 25.0 software. Categorical variables were presented in frequencies and continuous variables that showed a normal distribution were presented as the mean $\pm \mathrm{SD}$. When compared, paired samples $t$ test, ANOVA, or Mann-Whitney $U$ test was used to analyze the continuous indexes and the tests were two-sided, and the Chi square test was used to analyze categorical variables. $P<0.05$ was considered statistically significant.

\section{Results}

\section{General characteristics}

Age, region, health state, gestational week, educational background, and living condition are shown in Table 1 . The psychological susceptibility and the mood of pregnant women changes from the first trimester to the postpartum period. The distribution of the gestational weeks in the three different trimesters is shown in Table 2.

\section{SAS and SDS scores}

No significant changes were observed in SAS and SDS scores in different ages, regions, health states, gestational weeks, educational background, and living conditions $(P>0.05$, Table 1).

There were 79 cases $(50.6 \%, 79 / 156)$ of pregnant women with psychological health problem, of which $13(8.3 \%$, $13 / 156)$ suffered from anxiety, 79 patients $(50.6 \%, 79 / 156)$ suffered from depression, and 13 patients $(8.3 \%, 13 / 156)$ 
Table 1 SAS and SDS scores of pregnant women under different demographic characteristics

\begin{tabular}{|c|c|c|c|c|c|c|c|}
\hline Item & $n$ & SAS score & $\mathrm{F} / \mathrm{t}$ value & $P$ value & SDS score & $\mathrm{F} / \mathrm{t}$ value & $P$ value \\
\hline \multicolumn{8}{|l|}{ Age (year) } \\
\hline $20-25$ & 4 & $33.75 \pm 3.20$ & \multirow[t]{3}{*}{3.029} & \multirow[t]{3}{*}{$>0.05$} & $32.75 \pm 11.38$ & \multirow[t]{3}{*}{1.369} & \multirow[t]{3}{*}{$>0.05$} \\
\hline $26-30$ & 91 & $40.37 \pm 5.71$ & & & $40.51 \pm 8.96$ & & \\
\hline $31-50$ & 61 & $41.26 \pm 6.54$ & & & $40.57 \pm 9.64$ & & \\
\hline \multicolumn{8}{|l|}{ Region } \\
\hline Wuhan & 104 & $40.50 \pm 5.93$ & \multirow[t]{3}{*}{0.27} & \multirow[t]{3}{*}{$>0.05$} & $40.34 \pm 9.24$ & \multirow[t]{3}{*}{0.24} & \multirow[t]{3}{*}{$>0.05$} \\
\hline Hubei except Wuhan & 26 & $40.50 \pm 5.34$ & & & $39.42 \pm 9.05$ & & \\
\hline Not in Hubei & 26 & $40.80 \pm 7.48$ & & & $40.33 \pm 9.31$ & & \\
\hline \multicolumn{8}{|l|}{ Health state } \\
\hline Diagnosed/suspected & 11 & $39.45 \pm 9.05$ & \multirow[t]{2}{*}{-0.426} & \multirow[t]{2}{*}{$>0.05$} & $38.36 \pm 11.21$ & \multirow[t]{2}{*}{-0.727} & \multirow[t]{2}{*}{$>0.05$} \\
\hline Health & 145 & $40.63 \pm 5.84$ & & & $40.48 \pm 9.18$ & & \\
\hline \multicolumn{8}{|l|}{ Gestational week } \\
\hline $0 \sim 12$ week & 36 & $41.58 \pm 6.44$ & \multirow[t]{3}{*}{0.727} & \multirow[t]{3}{*}{$>0.05$} & $42.69 \pm 10.47$ & \multirow[t]{3}{*}{1.974} & \multirow[t]{3}{*}{$>0.05$} \\
\hline $13 \sim 24$ week & 46 & $40.48 \pm 6.44$ & & & $38.61 \pm 9.06$ & & \\
\hline $25 \sim 40$ week & 74 & $40.09 \pm 5.70$ & & & $40.26 \pm 8.74$ & & \\
\hline \multicolumn{8}{|l|}{ Educational background } \\
\hline High school/special secondary school & 23 & $40.83 \pm 4.06$ & \multirow[t]{4}{*}{1.149} & \multirow[t]{4}{*}{$>0.05$} & $41.78 \pm 7.78$ & \multirow[t]{4}{*}{0.885} & \multirow[t]{4}{*}{$>0.05$} \\
\hline Bachelor degree/College degree & 97 & $41.09 \pm 6.84$ & & & $40.62 \pm 10.14$ & & \\
\hline Master & 23 & $39.04 \pm 4.15$ & & & $39.70 \pm 8.14$ & & \\
\hline Doctor & 13 & $38.69 \pm 5.66$ & & & $36.77 \pm 6.72$ & & \\
\hline \multicolumn{8}{|l|}{ Live alone or not } \\
\hline Alone & 20 & $40.05 \pm 7.07$ & \multirow[t]{2}{*}{-0.393} & \multirow[t]{2}{*}{$>0.05$} & $41.20 \pm 9.74$ & \multirow[t]{2}{*}{0.445} & \multirow[t]{2}{*}{$>0.05$} \\
\hline Not alone & 136 & $40.62 \pm 5.96$ & & & $40.21 \pm 9.28$ & & \\
\hline
\end{tabular}

Table 2 Distribution of women at different gestational week in different region

Table 3 The comparison of mental health of pregnant women with or without COVID-19

\begin{tabular}{lr}
\hline Item & $n$ \\
\hline Wuhan & \\
$0 \sim 12$ week & 24 \\
$13 \sim 24$ week & 25 \\
$25 \sim 40$ week & 55 \\
Hubei except Wuhan & \\
$0 \sim 12$ week & 6 \\
$13 \sim 24$ week & 11 \\
$25 \sim 40$ week & 9 \\
Not in Hubei & \\
$0 \sim 12$ week & 6 \\
$13 \sim 24$ week & 10 \\
$25 \sim 40$ week & 10 \\
\hline
\end{tabular}

suffered from both anxiety and depression. The SAS score of the 156 subjects was $40.5513 \pm 6.09$, and the SDS score was $50.42 \pm 11.64$. For the SAS score, $91.67 \%(143 / 156)$ of the subjects were healthy individuals, while $8.3 \%(13 / 156)$ of the subjects were in a state of light anxiety. In the SDS score, $46.79 \%(73 / 156)$ of the subjects were healthy individuals, $23.72 \%(37 / 156)$ of patients suffered from a mild depression, $22.44 \%(35 / 156)$ of patients suffered from a moderate depression, and $4.49 \%$ (7/156) of patients suffered from a severe depression (Table 3 ).

Diagnosed/suspected patients with COVID-19 did not experience more severe anxiety or depression than individuals in the healthy control group $(P>0.05$, Table 3$)$. In addition, pregnant women in Wuhan did not experience more

\begin{tabular}{|c|c|c|c|c|c|c|}
\hline \multirow[t]{2}{*}{ Item } & \multicolumn{2}{|l|}{ SAS } & \multicolumn{4}{|l|}{ SDS } \\
\hline & Normal & Light & Normal & light & Medium & Heavy \\
\hline $\begin{array}{l}\text { Diagnosed/sus- } \\
\text { pected }\end{array}$ & 10 & 1 & 7 & 1 & 2 & 1 \\
\hline Health & 133 & 12 & 70 & 36 & 33 & 6 \\
\hline Total & 143 & 13 & 77 & 37 & 35 & 7 \\
\hline$\chi^{2}$ value & 0.009 & & 2.211 & & & \\
\hline$P$ value & $>0.05$ & & $>0.05$ & & & \\
\hline
\end{tabular}


Table 4 The comparison of mental health of pregnant women in Wuhan and nonWuhan regions

\begin{tabular}{|c|c|c|c|c|c|c|}
\hline \multirow[t]{2}{*}{ Item } & \multicolumn{2}{|l|}{ SAS } & \multicolumn{4}{|l|}{ SDS } \\
\hline & Normal & Light & Normal & light & Medium & Heavy \\
\hline Wuhan & 97 & 7 & 53 & 24 & 22 & 5 \\
\hline Non-Wuhan & 46 & 6 & 24 & 13 & 13 & 2 \\
\hline$\chi^{2}$ value & 1.049 & & 0.516 & & & \\
\hline$P$ value & $>0.05$ & & $>0.05$ & & & \\
\hline
\end{tabular}

severe anxiety or depression compared to pregnant women in non-Wuhan regions $(P>0.05$, Table 4$)$.

\section{Discussion}

Anxiety and depression is common in pregnant women. The detection rate of anxiety and depression varies in different countries and regions [19]. In general, about 4-15\% of pregnant women show symptoms of depression during pregnancy, $5-13 \%$ of pregnant women have anxiety symptoms, and in $0.9-3.8 \%$ of pregnant women depression and anxiety coexist [20]. According to the recent Chinese literature, during pregnancy, the level of anxiety in pregnant women accounts for $8.5-11.9 \%$, and the level of depression accounts for $3.5-8.2 \%$ [18, 21, 22]. According to our findings, the proportion of anxiety among pregnant women in China during the epidemic was $8.3 \%$, while the proportion of depression was $50.6 \%$. Furthermore, the proportion of moderate to severe depression reached $26.93 \%$. Thus, during the COVID-19 epidemic, the anxiety level of pregnant women was the same as that before the epidemic, while the level of depression was significantly higher.

COVID-19 patients had a high level of post-traumatic stress symptoms (PTSS) and a significantly higher level of depressive symptoms, while the general public had higher levels of anxiety and depression compared to before the COVID-19 epidemic, thereby indicating that the effects of the epidemic on the mental state are more apparent in making people depressed [23]. Moreover, in previous studies, it was suggested that the impact of depression during pregnancy was greater than that of anxiety [19]. Depression during pregnancy was not only associated with premature birth [24], but also increased the risk of low birth weight [25]. From the perspective of etiology, prenatal depression had a stronger impact on a low birth weight than on preterm birth [26]. Therefore, attention should be paid to the mental health of pregnant women, no exception during the COVID-19 epidemic, especially depression, and psychological counseling should be conducted in a timely manner.

It is generally thought that anxiety and depression are closely related, and many factors that can cause anxiety may also cause depression [19]. In terms of sociopsychological factors, stress and social support are the two factors with the greatest impact on pregnant women [19]. The occurrence of anxiety and depression in pregnant women is significantly related to stress [27], and pregnant women with a low social support system are more likely to develop anxiety and depression during pregnancy [28].

The factors affecting anxiety and depression are diverse and complex. Our results showed that part of the causes of depression and anxiety may be independent factors. In previous studies, it was shown that anxiety was linked to increased engagement in threat-avoidance behaviors, and that depression was associated with reduced engagement in reward-seeking behaviors [29, 30]. When the questionnaires were issued, the epidemic in China was in a stable period, more than a month after the temporarily shut down of Wuhan. At that time, the number of newly diagnosed cases in China gradually decreased, while the number of patients cured gradually increased. News reports during the epidemic showed China's high consideration and dedication to control COVID-19, and news reports mentioned the successful delivery of pregnant women with COVID-19 [31]. In addition, during the outbreak, family members of pregnant women were at home, which may increase the time spent with pregnant woman and increase social support.

Therefore, we speculated that transparent information, a stable epidemic situation, and increased social support may not increase the threat-avoidance behaviors of pregnant women with COVID-19, which may be the reason that the anxiety of pregnant women did not significantly increase during the epidemic. In contrast, measures of "shelter in place" caused pregnant women to have to live at home or be isolated during the epidemic. They were unable to undergo obstetric examinations and attend many social activities. The above-mentioned challenges may reduce engagement in reward-seeking behaviors and be the reason that pregnant women suffered from a severe depression during the epidemic.

Factors that affect the mental state of pregnant women are complex and multifaceted, and include age, educational background, occupation, gestational week, economic income, medical history, sociopsychological factors, etc. In this study, we showed that no significant difference was observed in SAS and SDS scores of pregnant women at different ages, regions, health state, gestational week, educational background, and living condition 
during the COVID-19 epidemic in China. Thus, pregnant women living in Wuhan, the epicenter of the COVID-19 epidemic in China, were not more anxious or depressed when compared to pregnant women in other regions during the COVID-19 epidemic, and the mental health of pregnant women with COVID-19 was not more severe. We speculated that the main reason for this may be that pregnant women were similarly isolated at home when the questionnaire was issued. In addition, the cost of treatment for COVID-19 patients in China was free at that stage of epidemic, which may be another reason.

Our study has some limitations. Although no significant differences were observed in SAS and SDS scores at different gestational weeks, the number of pregnant women at the postpartum stage was nearly twice as high compared to the number of pregnant women in the first or second trimester, which may affect the results.

At present, COVID-19 has been a global pandemic and many countries are experiencing the same rapid growth of confirmed cases. During the epidemic, the mental health of pregnant women is more vulnerable than usual. The psychological health of pregnant women is of utmost importance, and it should be paid attention to at any moment in time, not just during the epidemic. More attention should be paid to the mental health of pregnant women, especially depression, and timely guidance, intervention, and treatment should be provided. Rapid control of the epidemic, transparent information, and more social support would help protect the mental health of pregnant women. Furthermore, we recommend that more attention should be paid to the mental health status of the special group of pregnant women with COVID-19.

Author contributions HD: Manuscript writing, Project development; RH: Manuscript writing, Data analysis; CL: Data collection; DH: Data collection or management; DC: Data collection or management; GH: Manuscript polishing; MZ: Project development, Manuscript polishing.

Funding This work was supported by National Natural Science Foundation of China (No. 81803913) which granted to Haoxu Dong.

Code availability Not applicable.

\section{Compliance with ethical standards}

Conflict of interest The authors declare that they have no conflict of interest that could be perceived as prejudicing the impartiality of the research reported.

Ethics approval This study was approved by the Medical Ethics Committee of Tongji Hospital, Tongji Medical College, Huazhong University of Science and Technology (Approval number: TJ-IRB20200323).

Consent to participate All subjects orally agreed to participate in the study before completing the questionnaire.
Consent for publication All authors agree to contribute to this journal.

Availability of data and material The questionnaire was designed and published through the Questionnaire website (https://www.wjx.cn), and the original data can be checked on this website (ID:59179377).

\section{References}

1. Zhu N, Zhang D, Wang W et al (2019) A Novel coronavirus from patients with pneumonia in China. N Engl J Med 382:727-733

2. Baidu (2020)Epidemic real-time big data report. Baidu website. https://voice.baidu.com/act/newpneumonia/newpneumon ia/?from=osari_pc_3. (Accessed 27 Mar 2020).

3. WHO (2020) Coronavirus disease 2019 (COVID-19)situation report-85.WHO website https://www.who.int/emergencies/disea ses/novel-coronavirus-2019/situation-reports/. (Accessed 14 Apr 2020).

4. Jamieson DJ, Theiler RN, Rasmussen SA (2020) Emerging infections and pregnancy. Emerg Infect Dis 12:1638-2164

5. Wong SF, Chow KM, de Swiet M (2003) Severe acute respiratory syndrome and pregnancy. BJOG 110:641-642

6. Wong SF, Chow KM, Leung TN et al (2004) Pregnancy and perinatal outcomes of women with severe acute respiratory syndrome. Am J Obstet Gynecol 191:292-297

7. Ng PC, So KW, Leung TF et al (2003) Infection control for SARS in a tertiary neonatal center. Arch Dis Child Fetal Neonatal Ed 88:F405-F409

8. Schwartz DA, Graham AL (2020) Potential maternal and infant outcomes from (Wuhan) Coronavirus 2019-nCoV infecting pregnant women: lessons from SARS, MERS, and other human coronavirus infections. Viruses 12(2):194

9. Harville EW, Xiong X, Pridjian G et al (2009) Postpartum mental health after Hurricane Katrina: a cohort study. BMC Pregnancy Childbirth 9:21

10. Engel SM, Berkowitz GS, Wolff MS et al (2005) Psychological trauma associated with the world trade center attacks and its effect on pregnancy outcome. Paediatr Perinat Epidemiol 19:334-341

11. Harville EW, Xiong X, Buekens P et al (2010) Resilience after Hurricane Katrinaamong pregnant and postpartum women. Women's Health Iss 20:20-27

12. Khatri GK, Tran TD, Baral S et al (2018) Effect of the 2015 Nepal Earthquakes on symptoms of common mental disorders among women who are pregnant. J Affect Disord 228:238-247

13. Sade S, Sheiner E, Wainstock T et al (2020) Risk for depressive symptoms among hospitalized women in high-risk pregnancy units during the COVID-19 Pandemic. J Clin Med 9(8):2449

14. Leung KF, Liu FB, Zhao L et al (2005) Development and validation of the Chinese Quality of Life Instrument. Health Qual Life Outcomes 3:26

15. Zung WW, Maqruder-habib K, Velez R et al (1990) The comorbidity of anxiety and depression in general medical patients: a longitudinal study. J Clin Psychiatry 51:77-80

16. Jokelainen J, Timonen M, Keinänen-Kiukaanniemi S et al (2019) Validation of the Zung self-rating depression scale (SDS) in older adults. Scand J Prim Health Care 37(3):353-357

17. Mormont E, Jamart J, Jacques D (2014) Symptoms of depression and anxiety after the disclosure of the diagnosis of Alzheimer disease. J Geriatr Psychiatry Neurol 27:231-236

18. Mao HF, Rong HH, Wang ZW et al (2017) Survey on changes in mental health and health needs of pregnant women in Jiading District of Shanghai. Mater Child Health Care Chin 32(12):2729-2734 
19. Ma XM, Shi HJ (2019) Research progress on anxiety and depression levels and influencing factors in pregnant women. Mater Child Health Care Chin 34(03):722-724

20. Brunton RJ, Dryer R, Saliba A et al (2015) Pregnancy anxiety: a systematic review of current scales. J Affect Disord 176(1):24-34

21. Hu HQ, Zhang J, Zhao W et al (2017) The occurrence and determinants of anxiety and depression symptoms in women of six counties/districts in China during pregnancy. Chin J Prev Med 51(1):47-52

22. Zheng YY, Sun Y, Liu XW (2019) Investigation of anxiety and depression status in late pregnancy and analysis of related factors. Chin J Prev Med 34(07):1631-1633

23. Vindegaard N, Benros ME (2020) COVID-19 pandemic and mental health consequences: systematic review of the current evidence. Brain Behav Immun S091591(20):30954-30955 (published online ahead of print, 2020 May 30)

24. Pearson RM, Evans J, Kounali D et al (2013) Maternal depression during pregnancy and the postnatal period: risks and possible mechanisms for offspring depression at age 18 years. JAMA Psychiatry 70(12):1312-1319

25. Verbeek T, Arjadi R, Vendrik JJ et al (2015) Anxiety and depression during pregnancy in Central America: a cross-sectional study among pregnant women in the developing country Nicaragua. BMC Psychiatry 15(1):292

26. Vollrath ME, Sengpiel V, Landolt MA et al (2016) Is maternal trait anxiety a risk factor for late preterm and early term deliveries? BMC Pre Childbirth 16(1):286
27. Basharpoor S, Heydarirad H, Daryadel SJ et al (2017) The role of perceived stress and social support among predicting anxiety in pregnant women. J Holist Nurs Midwifery 27(2):9-16

28. Van Heyningen T, Honikman S, Myer L et al (2017) Prevalence and predictors of anxiety disorders amongst low-income pregnant women in urban South Africa: a cross-sectional study. Arch Women's Ment Healt 20(6):765-775

29. Sonia J, Gagn BC (2018) Anxiety, depression, and decision making: a computational perspective. Annu Rev Neurosci 41:371-388

30. Li M, D'Arcy C, Meng X (2016) Maltreatment in childhood substantially increases the risk of adult depression and anxiety in prospective cohort studies: systematic review, meta-analysis, and proportional attributable fractions. Psychol Med 46:717-730

31. Changjiang Daily (2020) The first case in Hubei! New pneumonia in Wuhan is highly suspected to have a successful baby. Changjiang Daily website https://m.top.cnr.cn/sogoudh/wzy/20200125/ t20200125_524949228.html. (Accessed 25 Janu 2020).

Publisher's Note Springer Nature remains neutral with regard to jurisdictional claims in published maps and institutional affiliations. 